\title{
Was wissen die Bürger über Politik? Zur Erforschung der politischen Kenntnisse in der Bundesrepublik Deutschland 1949 bis 2008
}

\author{
Jürgen Maier, Alexander Glantz und Severin Bathelt
}

\section{Die politischen Kenntnisse der Bürger: Kein Thema für die deutsche Politikwissenschaft}

Wissenstests sind in Deutschland zurzeit in Mode: Die Fernsehsender überbieten sich gegenseitig mit der Ausstrahlung von Quizshows (zum Beispiel ARD: „Das Quiz mit Jörg Pilawa“, RTL: „Wer wird Millionär?“). Im Internet - etwa bei den Online-Ausgaben von Tageszeitungen - kursieren zahllose Angebote, in denen die Nutzer ihre Kenntnisse über die verschiedensten Themen auf den Prüfstand stellen können. Selbst die Bundesländer sind flächendeckend dazu übergegangen, einbürgerungswillige Ausländer daraufhin zu testen, ob sie das „für das Bekenntnis zum Grundgesetz notwendige Verständnis von Prinzipien und Aufbau des deutschen Staatswesens sowie der damit in Zusammenhang stehenden gesellschaftspolitischen Prozesse“ parat haben und damit „ihre staatsbürgerlichen Rechte und Pflichten erkennen und aktiv wahrnehmen zu können"1. Man könnte also den Eindruck gewinnen, die Deutschen seien durchweg hochinformiert und würden keine Gelegenheit auslassen, ihre Kenntnisse über die Welt einem Belastungstest auszusetzen - und deshalb auch diejenigen, die die deutsche Staatsbürgerschaft anstreben, gewissenhaft daraufhin überprüfen werden, ob ihr Wissensniveau zumindest hinsichtlich politischer Fragen gewisse Mindeststandards nicht unterschreitet.

\subsection{Der Forschungsstand in den USA}

Für die Annahme, die Bundesbürger wären gut informiert, spricht allerdings wenig. Dies gilt zumindest für den Bereich der Politik. Zwar gibt es zu diesem Thema nur erstaunlich wenig gesicherte - und an späterer Stelle noch zu berichtende - empirische Befunde. Doch warum sollten hierzulande die Dinge anders gelagert sein als in anderen westlichen Demokratien? Vor allem in den Vereinigten Staaten beschäftigt sich die politikwissenschaftliche Forschung schon sehr lange mit der Frage, was Bürger über Politik wissen. Mit der Durchführung von flächendeckenden Bevölkerungsbefragungen wurden die düsteren Vorahnungen früherer Jahre ${ }^{2}$ auch empirisch bestätigt: „The democratic citizen is expected to be well informed about political affairs. He is supposed to know what the issues are, what their history is, what the relevant facts are, what alternatives are proposed, what the party stands for, what the likely consequences are. By such standards the voter falls short." 3

1 Anlage 2 zur Einbürgerungstestverordnung; http://www.bmi.bund.de/cae/servlet/contentblob/123032/publicationFile/13218/Rahmencurriculum.pdf (Abruf am 21. Juni 2009).

2 Vgl. James Bryce, The American Commonwealth, Vol. 3, London 1888, S. 8; Walter Lippman, Public Opinion, New York 1922; ders., The Phantom Republic, New York 1925.

3 Bernard R. Berelson / Paul F. Lazarsfeld / William N. McPhee, Voting: A Study of Opinion Formation in a Presidential Campaign, Chicago 1954, S. 308. 
Der von Berelson, Lazarsfeld und McPhee 1954 formulierte Eindruck verdichtete sich schnell. Gestützt auf die Ergebnisse zahlreicher empirischer Untersuchungen ${ }^{4}$ war sich die Politikwissenschaft knapp drei Jahrzehnte später einig, dass die Verteilung von politischem Wissen in der Bevölkerung einer klaren, über die USA hinausgehenden Gesetzmäßigkeit folgt. So etwa stellte Converse fest: „The two simplest truths I know about the distribution of political information in modern electorates are that the mean is low and the variance is high. "5 Page und Shapiro konstatierten im selben Zeitraum, es sei ein „well-established finding that most peoples' knowledge of politics is quite meager"6. Und Luskin befand: „There now seems to be a near-consensus that by anything approaching elite standards most citizens think and know jaw-droppingly little about politics."7

In jüngerer Zeit wurden aber auch gemäßigtere Urteile über die politischen Kenntnisstände der Bürger gefällt. So etwa stellten Delli Carpini und Keeter fest: „Although citizens are not fully informed, they are not fully ignorant. " ${ }^{8}$ Diese Einschätzung gründet einerseits auf der Beobachtung, dass sich beim Bürger nicht nur Wissenslücken beobachten lassen, sondern es auch Bereiche gibt, in denen sich die Bürger gut auskennen: So etwa wissen 99

4 Vgl. zum Beispiel Gabriel A. Almond / Sidney Verba, The Civic Culture. Political Attitudes and Democracy in Five Nations, Princeton 1963; Stephen E. Bennett, Know-Nothings Revisited: The Meaning of Political Ignorance Today, in: Social Science Quarterly, 69. Jg. (1988), H. 2, S. 476 - 490; ders., Trends in Americans' Political Information, 1967-1987, in: American Politics Quarterly, 17. Jg. (1989), H. 4, S. 422 - 435; Stephen E. Bennett / Richard S. Flickinger / John R. Baker / Stacy L. Rhine u.a., Citizens' Knowledge of Foreign Affairs, in: Harvard International Journal of Press/Politics, 1. Jg. (1996), H. 2, S. 10 - 29; Phillip E. Converse, The Nature of Belief Systems in Mass Publics, in: David E. Apter (Hrsg.), Ideology and Discontent, New York 1964, S. 206 261; Phillip E. Converse, Public Opinion and Voting Behavior, in: Fred I. Greenstein / Nelson W. Polsby (Hrsg.), Handbook of Political Science, Vol. 4, Reading 1975, S. 75 - 169; Michael X. Delli Carpini / Scott Keeter, Stability and Change in the U.S. Public's Knowledge of Politics, in: Public Opinion Quarterly, 55. Jg. (1991), S. 583 - 612; dies., What Americans Know about Politics and Why it Matters, New Haven 1996; Michael A. Dimock, / Samuel L. Popkin, Political Knowledge in Comparative Perspective, in: Shanto Iyengar / Richard Reeves (Hrsg.), Do the Media Govern? Politicians, Voters, and Reporters in America, Thousand Oaks 1997, S. 217 - 224; Hazel G. Erskine, The Polls: The Informed Public, in: Public Opinion Quarterly, 26. Jg. (1962), S. 668 - 677; dies., The Polls: Textbook Knowledge, in: Public Opinion Quarterly, 27. Jg. (1963), S. 133 - 141; dies., The Polls: Exposure to Domestic Information, in: Public Opinion Quarterly, 27. Jg. (1963), S. 491 - 500; dies., The Polls: Exposure to International Information, in: Public Opinion Quarterly, 27. Jg. (1963), S. 65 - 662; John A. Ferejohn, Information and the Electoral Process, in: ders. I James H. Kuklinski (Hrsg.), Information and Democratic Processes, Urbana 1990, S. 1 - 19; Robert E. Lane / David O. Sears, Public Opinion, Englewood Cliffs 1964; W. Russell Neuman, The Paradox of Mass Politics. Knowledge and Opinion in the American Electorate, Cambridge 1986; E.R.A.N. Smith, The Unchanging American Voter, Berkeley 1989.

5 Vgl. Phillip E. Converse, Popular Representation and the Distribution of Political Information, in: John A. Ferejohn / James H. Kuklinski (Hrsg.), a.a.O. (Fn. 4), S. 372; ähnlich auch Phillip E. Converse, Assessing the Capacity of Mass Electorates, in: Annual Review of Political Science, 3. Jg. (2000), S. $331-353$.

6 Vgl. Benjamin I. Page / Robert Y. Shapiro, The Rational Public: Fifty Years of Trends in Americans' Policy Preferences, Chicago 1992, S. 9.

7 Vgl. Robert C. Luskin, From Denial to Extenuation (and Finally Beyond). Political Sophistication and Citizen Performance, in: James H. Kuklinski (Hrsg.), Thinking About Political Psychology, Cambridge 2002, S. 281 - 305, S. 282.

8 Vgl. Michael X. Delli Carpini / Scott Keeter, What Americans Know About Politics and Why it Matters, a.a.O. (Fn. 4), S. 98. 
Prozent der Amerikaner, wer ihr Präsident ist und 96 Prozent, dass die Vereinigten Staaten Mitglied der UNO sind. ${ }^{9}$ Hinzu kommt, dass man in der US-Forschung zwischenzeitlich nicht nur auf das absolute Wissensniveau abstellt, sondern sich verstärkt mit den Ursachen (sowie den Konsequenzen) fehlender Politikkenntnisse beschäftigt. ${ }^{10}$

Einen wesentlichen Beitrag zu einer differenzierteren Sichtweise hat hier die Politische Psychologie geleistet, deren Einblicke in die menschliche Informationsaufnahme zu einem besseren Verständnis geführt haben, warum Bürger so wenig über die Welt der Politik wissen - und warum dies kein Problem darstellen muss. ${ }^{11}$ Wesentlich in diesem Zusammenhang sind folgende Erkenntnisse: Erstens können Menschen schon aufgrund begrenzter Verarbeitungskapazitäten nicht alle Informationen aufnehmen - selbst wenn sie wollten. Vielmehr muss eine Auswahl unter den zur Verfügung stehenden Informationen getroffen werden, die in aller Regel unbewusst stattfindet. Zweitens bindet jegliche Auseinandersetzung mit Informationen Ressourcen. Dieser Prozess ist also kostspielig - ebenso wie die normativ erwünschte, aktive Suche nach Neuigkeiten. Umgekehrt kann aber der Grenznutzen zusätzlicher Informationen sehr gering sein, so dass ab einem bestimmten Wissensniveau die Verarbeitung neuer Hinweise unattraktiv ist. Drittens können Bürger offenbar auch sehr gut ohne umfassendes Wissen über politische Zusammenhänge - und damit relativ „kostengünstig“ - zu vernünftigen Bewertungen und Entscheidungen kommen. ${ }^{12}$ Verwendet werden in diesem Zusammenhang so genannte „information shortcuts“. Der erwartete Inhalt einer Information wird dabei anhand einfacher Heuristiken erschlossen. So etwa reicht für parteigebundene Wähler ein Hinweis darauf, welche Partei einen Vorschlag zur Lösung eines Problems gemacht hat, völlig aus, um zu beurteilen, was von diesem zu halten ist.

Über diese kognitionspsychologischen Erklärungen hinaus, die einerseits für eine Relativierung, andererseits für eine weniger durch normative Sichtweisen geprägte Diskussion über die begrenzten Politikkenntnisse der Bürger sorgt, ist in der internationalen Fachliteratur zwischenzeitlich auch eine heftige Methodendebatte entbrannt, die um die Frage kreist, welche Eigenschaften Instrumente haben müssen, mit denen man versucht, politisches Wissen zu erfassen. Ein Thema ist dabei auch, ob die in diesem Zusammenhang verwendeten Datenerhebungstechniken - insbesondere standardisierte Telefon- und Face-toFace-Befragungen - nicht dazu beitragen, dass die politischen Kenntnisstände der Bürger systematisch unterschätzt werden. ${ }^{13}$ Auch wenn diese Diskussion noch nicht abgeschlossen und demnach der Gehalt verschiedener empirischer Befunde nicht endgültig bewertet werden kann, sorgt die Forschung auf diesem Gebiet dafür, dass die Sensibilisierung für methodische Fragestellungen wächst. Festgehalten werden kann schon jetzt, dass die in Umfragen gemessenen niedrigen Wissensstände nicht nur ein Beleg für die - je nach Blick-

9 Ebenda, S. 70, 74.

10 Vgl. zum Beispiel ebenda.

11 Vgl. zusammenfassend zum Beispiel David O. Sears / Leonie Huddy / Robert Jervis (Hrsg.), Oxford Handbook of Political Psychology, Oxford 2003.

12 Vgl. zum Beispiel Daniel Kahneman / Paul Slovic / Amos Tversky, Judgement Under Uncertainty: Heuristics and Biases, New York 1982; Richard E. Nisbett / Lee Ross, Human Inference: Strategies and Shortcomings of Social Judgment, Englewood Cliffs 1980.

13 Vgl. zum Beispiel Markus Prior / Arthur Lupia, Money, Time, and Political Knowledge. Distinguishing Quick Recall and Political Learning Skills, in: American Journal of Political Science, 52. Jg. (2008), H. 1, S. $169-183$. 
winkel - mehr oder weniger ausgeprägte politische Ignoranz der Bürger, sondern auch ein Produkt der verwendeten Methoden sind.

\subsection{Der Forschungsstand in Deutschland}

Auch wenn in den USA immer wieder angemahnt wird, dass die Forschungen auf diesem Gebiet verstärkt werden müssen ${ }^{14}$, liegt dort bereits eine Vielzahl differenzierter Befunde über das politische Wissen der Bürger vor. Hingegen ist sehr wenig bekannt über die Verteilung, die Entwicklung, die Strukturen, die Determinanten und die Konsequenzen (einerseits für den Bürger, andererseits für das politische System) von (fehlenden) Politikkenntnissen in Deutschland. Tatsächlich liegt nur eine Handvoll wissenschaftlicher und für die Bevölkerung repräsentativer Untersuchungen vor. Das, was wir über die politischen Kognitionen der Deutschen wissen, ist vielfach nur ein Abfallprodukt von Forschungen zu völlig anderen Themen. Auch in deutschsprachigen Standardwerken zur Politischen Soziologie, zur Wahl- und Einstellungsforschung oder zur Politischen Kommunikation fehlen eigenständige Beiträge zu politischem Wissen ${ }^{15}$; häufig sind nicht einmal im Stichwortverzeichnis Hinweise auf entsprechende Textstellen im Buch zu finden.

Die meisten Erkenntnisse liegen noch zur Verteilung politischer Kenntnisse vor. Dabei ist immer wieder zu erkennen, dass das objektiv vorhandene Wissen über wichtige institutionelle Strukturen ${ }^{16}$, die Funktionsweise des Wahlsystems ${ }^{17}$, den föderalen Aufbau der Bundesrepublik ${ }^{18}$, internationale Akteure wie die EU oder die NATO ${ }^{19}$ oder die ideologi-

14 Vgl. W. Russell Neuman, a.a.O. (Fn. 4), S. 8.

15 Eine Ausnahme ist die Monographie von Winfried Schulz, Politische Kommunikation: Theoretische Ansätze und Ergebnisse empirischer Forschung, Wiesbaden 2008.

16 Vgl. Werner J. Patzelt, Warum verachten die Deutschen ihr Parlament und lieben ihr Verfassungsgericht? Ergebnisse einer vergleichenden demoskopischen Studie, in: ZParl, 36. Jg. (2005), H. 3, S. 517 - 538; Suzanne S. Schüttemeyer, Bundestag und Bürger im Spiegel der Demoskopie, Opladen 1986.

17 Vgl. zum Beispiel Eckhard Jesse, Split-Voting in the Federal Republic of Germany: An Analysis of the Federal Elections from 1953 to 1987, in: Electoral Studies, 7. Jg. (1988), H. 2, S. 109 - 124; Jeffrey A. Karp, Political Knowledge about Electoral Rules: Comparing Mixed Member Proportional Systems in Germany and New Zealand, in: Electoral Studies, 25. Jg. (2006), H. 4, S. 714 - 730; Jürgen Maier, Politisches Interesse und politisches Wissen in Ost- und Westdeutschland, in: Jürgen W. Falter / Oscar W. Gabriel / Hans Rattinger (Hrsg.), Wirklich ein Volk? Die politischen Orientierungen von Ost- und Westdeutschen im Vergleich, Opladen 2000, S. 141 - 171; Rüdiger Schmitt-Beck, Denn sie wissen nicht, was sie tun ... Zum Verständnis des Verfahrens der Bundestagswahl bei westdeutschen und ostdeutschen Wählern, in: ZParl, 24. Jg. (1993), H. 3, S. 393 - 414; Suzanne S. Schüttemeyer, a.a.O. (Fn. 16); Angelika Vetter / Jürgen Maier, Mittendrin statt nur dabei? Politisches Wissen, politisches Interesse und politisches Kompetenzgefühl in Deutschland, in: Oscar W. Gabriel / Jürgen W. Falter / Hans Rattinger (Hrsg.), Wächst zusammen, was zusammengehört? Stabilität und Wandel politischer Einstellungen im wiedervereinigten Deutschland, Baden-Baden 2005, S. 51 - 90; Bettina Westle, Politisches Wissen und Wahlen, in: Jürgen W. Falter / Oscar W. Gabriel / Bernhard Weßels (Hrsg.), Wahlen und Wähler: Analysen aus Anlass der Bundestagswahl 2002, Wiesbaden 2005, S. 484 - 512.

18 Vgl. Jürgen Maier, a.a.O. (Fn. 17).

19 Vgl. zum Beispiel Hans Rattinger, Public Attitudes to European Integration in Germany after Maastricht: Inventory and Typology, in: Journal of Common Market Studies, 32. Jg. (1994), H. 4, S. $525-540$. 
schen beziehungsweise sachpolitischen Positionen der Parteien ${ }^{20}$ begrenzt ist. Ost-WestVergleiche zeigen, dass politische Wissensstände in den neuen Bundesländern tendenziell geringer ausfallen als in den alten Ländern, wenngleich diese Differenzen zunehmend verschwinden. Daher liegt es nahe, Ost-West-Unterschiede darauf zurückzuführen, dass Bürger in den neuen Bundesländern auf einen geringeren Erfahrungsschatz im Umgang mit dem politischen System zurückgreifen können - und deshalb weniger über seine Strukturen, Akteure und Ergebnisse wissen. ${ }^{21}$ Die raren international vergleichend angelegten Studien zeigen zudem, dass es um die politische Urteilsfähigkeit der deutschen Wähler vergleichsweise gut bestellt ist. ${ }^{22}$

Die wenigen Untersuchungen, die die Verteilung von politischem Wissen im Längsschnitt unter die Lupe nehmen, belegen, dass politische Kenntnisse im Aggregat über die Zeit hinweg relativ stabil verteilt sind ${ }^{23}$ - und zwar obwohl Deutschland (ebenso wie andere westliche Demokratien) einem umfassenden Strukturwandel unterworfen ist, der sich potenziell positiv auf das Wissensniveau der Bevölkerung niederschlagen sollte (zum Beispiel die Erhöhung des Bildungsstandes oder die Ausweitung des von den Massenmedien bereitgestellten Informationsangebots). Kurzfristige Dynamiken können allerdings im Umfeld von Wahlen nachgewiesen werden: Politische Kenntnisse wachsen im Laufe des Wahlkampfs an und liegen nach einer Wahl über den zu Beginn des Wahlkampfs ermittelten Werten. ${ }^{24}$ Demgegenüber zeigen Panelstudien jedoch, dass die Stabilität von politischen Kenntnissen deutlich überschätzt wird. So liegt zum Beispiel der Anteil derjenigen Bürger, die zwischen den Bundestagswahlen 1998 und 2002 ein unverändertes Wissen über Politik aufwiesen, bei nur etwa einem Drittel. Ein weiteres Drittel konnte sein Urteilsvermögen in diesem Zeitraum verbessern; das restliche Drittel hat Kenntnisse eingebüßt. ${ }^{25}$

Während die Struktur politischen Wissens in Deutschland noch völlig unerforscht ist, sind die Erkenntnisse, die zu den Determinanten (fehlender) Politikkenntnisse vorliegen, umfangreicher. Die stärksten Effekte auf den Umfang politischer Kognitionen werden in aller Regel für das Geschlecht, den formalen Bildungsgrad, die Parteibindung, das politische Interesse und das subjektive Kompetenzgefühl ermittelt. ${ }^{26}$ Weniger systematisch sind die Wirkungen von Wertorientierungen, Schichtzugehörigkeit, Indikatoren sozialer Integration (Kirchenbindung, Gewerkschaftsmitgliedschaft, Parteimitgliedschaft) oder Persönlichkeitseigenschaften (zum Beispiel Anomie). ${ }^{27}$ Unerwartet schwache Einflüsse zeigen sich

20 Vgl. Angelika Vetter / Jürgen Maier, a.a.O. (Fn. 17); Bettina Westle, a.a.O. (Fn. 17).

21 Vgl. Jeffrey A. Karp, a.a.O.; Jürgen Maier, a.a.O. (Fn. 17); Rüdiger Schmitt-Beck, a.a.O. (Fn. 17); Bettina Westle, a.a.O. (Fn. 17); Angelika Vetter / Jürgen Maier, a.a.O. (Fn. 17).

22 Vgl. zum Beispiel Gabriel A. Almond / Sidney Verba, a.a.O. (Fn. 4); Michael X. Delli Carpini I Scott Keeter, What Americans Know About Politics and Why it Matters, a.a.O. (Fn. 4); Michael A. Dimock / Samuel L. Popkin, a.a.O. (Fn. 4); Jeffrey A. Karp, a.a.O. (Fn. 17).

23 Vgl. Jeffrey A. Karp, a.a.O. (Fn. 17); Angelika Vetter / Jürgen Maier, a.a.O. (Fn. 17).

24 Vgl. Jeffrey A. Karp, a.a.O. (Fn. 17); Jürgen Maier, a.a.O. (Fn. 17); Rüdiger Schmitt-Beck, a.a.O. (Fn. 17); Klaus Schönbach, Das unterschätzte Medium. Politische Wirkungen von Presse und Fernsehen im Vergleich, München 1983.

25 Vgl. Angelika Vetter / Jürgen Maier, a.a.O. (Fn. 17); ähnlich auch Rüdiger Schmitt-Beck für die Bundestagswahl 1990, a.a.O. (Fn. 17).

26 Vgl. Rüdiger Schmitt-Beck, a.a.O. (Fn. 17); Jürgen Maier, a.a.O. (Fn. 17); Winfried Schulz, a.a.O. (Fn. 15); Angelika Vetter / Jürgen Maier, a.a.O. (Fn. 17); Bettina Westle, a.a.O. (Fn. 17).

27 Vgl. Jürgen Maier, a.a.O. (Fn. 17); Angelika Vetter / Jürgen Maier, a.a.O. (Fn. 17). 
hinsichtlich der Mediennutzung. ${ }^{28}$ Tendenziell wirkt sich die Nutzung von Qualitätsmedien (insbesondere von Zeitungen) positiv auf das politische Urteilsvermögen aus, während der Konsum von Boulevardmedien negativ mit politischen Kenntnissen zusammenhängt. ${ }^{29}$ Experimentaluntersuchungen zeigen allerdings, dass die Rezeption bestimmter Fernsehformate wie etwa der so genannten TV-Duelle durchaus zu erheblichen und kurzfristigen Wissenszuwächsen führt. ${ }^{30}$ Dies gilt - allerdings mit größeren Einschränkungen - auch für Wahlwerbesendungen. ${ }^{31}$

Empirische Hinweise, dass (fehlende) Politikkenntnisse Konsequenzen für politische Einstellungen und Verhaltensabsichten haben, liegen für Deutschland nur vereinzelt vor. Diese konzentrieren sich zudem auf die Frage, ob die Kenntnis des Wahlsystems taktisches Wahlverhalten fördert. Die vorliegenden Ergebnisse sind hier tendenziell negativ: Wähler, die die Funktion von Erst- und Zweitstimme kennen, machen nicht häufiger von der Möglichkeit des Stimmensplittings Gebrauch als Wähler, denen die Bedeutung der ihnen zur Verfügung stehenden Stimmen unklar ist. ${ }^{32}$ Individuelle Unterschiede in der Verteilung politischer Kenntnisse in Deutschland bleiben offenbar also ohne größere Folgen für das Wahlergebnis - zumindest dann, wenn Wahlen deutlich entschieden werden und die aufgrund der Unkenntnis des Wahlsystems entgegen den eigenen Präferenzen - und damit „falsch“ - gesplitteten Stimmen ohne Bedeutung für den Ausgang sind. ${ }^{33}$ Bestimmte Trends im Wahlverhalten - insbesondere das Abschmelzen der Parteibindung und die damit einhergehende verstärkte Neigung zur Wechselwahl sowie die Tendenz zu knapperen Ergebnissen - indizieren aber, dass sich dieses aus demokratietheoretischer Sicht bislang irrelevante Problem nicht dauerhaft so darstellen muss.

Insgesamt kann also festgehalten werden, dass - besonders im Unterschied zu den USA - nur sehr wenige empirisch abgesicherte Erkenntnisse über das politische Wissen der Bundesbürger vorliegen. Und ob diese schmalen Befunde generalisierbar sind, ist fraglich, denn erstens konzentrieren sich die Studien, die sich explizit mit den Ursachen und Wirkungen politischer Kenntnisse auseinandersetzen, auf den Zeitraum nach 1990. Eine Über-

28 Vgl. zusammenfassend Jürgen Maier, Medienrezeption und politisches Wissen - ein Überblick über den Forschungsstand, in: Barbara Pfetsch / Frank Marcinkowski (Hrsg.), Politik in der Mediendemokratie, Wiesbaden 2009.

29 Vgl. Jürgen Maier, a.a.O. (Fn. 17); Rüdiger Schmitt-Beck, a.a.O. (Fn. 17); Winfried Schulz, a.a.O. (Fn. 15); Angelika Vetter / Jürgen Maier, a.a.O. (Fn. 17); Bettina Westle, a.a.O. (Fn. 17).

30 Vgl. zum Beispiel Jürgen Maier, Eine Basis für rationale Wahlentscheidungen? Die Wirkung des TV-Duells auf politische Kenntnisse, in: Marcus Maurer / Carsten Reinemann / Jürgen Maier / Michaela Maier, Schröder gegen Merkel: Wahrnehmung und Wirkung des TV-Duells im OstWest-Vergleich, Wiesbaden 2007, S. 129 - 143; Michaela Maier, Viel Spielraum für die eigene Interpretation. Wahrnehmung und Wirkung der Nachberichterstattung, ebenda, S. 195 - 227; Marcus Maurer / Carsten Reinemann, Schröder gegen Stoiber: Nutzung, Wahrnehmung und Wirkung der TV-Duelle, Wiesbaden 2003.

31 Vgl. Michaela Maier / Jürgen Maier, Nebensache Europa. Parteienspots zur Europawahl 2004 und ihre Wirkung - Ergebnisse einer Experimentalstudie, in: Jens Tenscher (Hrsg.), Wahl-Kampf um Europa. Analysen aus Anlass der Wahlen zum Europäischen Parlament 2004, Wiesbaden 2005, S. $118-135$.

32 Vgl. Jeffrey A. Karp, a.a.O. (Fn. 17); Bettina Westle, a.a.O. (Fn. 17); anders: Harald Schoen, Ist Wissen auch an der Wahlurne Macht? Politische Kompetenz und Wahlverhalten, in: Siegfried Schumann / Harald Schoen (Hrsg.), Persönlichkeit. Eine vergessene Größe der empirischen Sozialforschung, Wiesbaden 2005, S. 137 - 155.

33 Vgl. Rüdiger Schmitt-Beck, a.a.O. (Fn. 17). 
tragung auf frühere Phasen ist aufgrund der erheblichen Veränderungen der gesellschaftlichen, politischen und medialen Rahmenbedingungen in den letzten Jahrzehnten mit großen Unsicherheiten verbunden. Gleiches gilt für die Anwendung der US-amerikanischen Befunde auf deutsche Verhältnisse. Auch hier sind die System- und Kulturunterschiede zu groß. ${ }^{34}$ Zweitens arbeiten die meisten deutschen Studien mit Daten, die zeitnah zu Bundestagswahlen erhoben wurden. Aufgrund der (unter anderem auch kognitiv) mobilisierenden Wirkung von Wahlkämpfen besteht mit der Übertragung der vorliegenden Ergebnisse auf Zeiten, in denen keine nationalen Wahlkämpfe stattfinden, die Gefahr, dass das Wissensniveau der Bundesbürger systematisch überschätzt wird. Drittens fokussieren zahlreiche der Untersuchungen, die sich explizit mit der Rolle politischer Expertise auseinandersetzen, auf einen sehr begrenzten Satz von Wissensfragen und insbesondere auf die Kenntnis des Wahlsystems. Möglicherweise stellt sich aber die Situation in anderen Politikbereichen völlig anders dar. Viertens wird der Begriff „politisches Wissen“ in der Literatur nicht nur sehr unterschiedlich verstanden, sondern auch höchst unterschiedlich operationalisiert. Populär sind auch Ansätze, die Fragen zu politischem Wissen mit anderen Fragen zum Beispiel nach dem Politikinteresse - verknüpfen. Ziel ist es dabei, einen breit gefassten Indikator für die „politische Sophistizierung“ von Bürgern zu generieren. ${ }^{35}$ Vergleiche zwischen den Ergebnissen verschiedener Studien sind deshalb nur begrenzt möglich. Fünftens variieren die methodischen Standards erheblich, die in den verschiedenen Untersuchungen angelegt werden. Dies betrifft insbesondere die Frage nach Zusammenhängen zwischen Determinanten und Effekten (fehlender) Politikkenntnisse, die häufig nur bivariat - und damit ohne Kontrolle relevanter Variablen - berichtet werden.

Es ist erstaunlich, dass das Thema "politisches Wissen“ in Deutschland - im Gegensatz zur großen Beachtung in anderen Ländern - kaum Resonanz erfährt. Eine Ursache könnte darin liegen, dass die in der amerikanischen Politikwissenschaft zu beobachtende „kognitive Wende ${ }^{\text {" } 36}$ noch nicht in Deutschland angekommen ist. ${ }^{37}$ Erste Bemühungen, politische Wissensstände als Erklärungsgröße in empirische Untersuchungen aufzunehmen oder sogar dezidiert zum Gegenstand der Analysen zu machen, zeigen sich noch am ehesten in der Wahl- und Politischen Kommunikationsforschung. Zweitens ist zu vermuten, dass politisches Wissen mangels adäquater Daten in empirischen Untersuchungen ausgeblendet wird.

Im vorliegenden Beitrag soll anhand der in Deutschland verfügbaren Umfragedaten untersucht werden, wie es um das Politikwissen der Bundesbürger bestellt ist ${ }^{38}$ : Erstens, gibt

34 Vgl. Michael A. Dimock / Samuel L. Popkin, a.a.O. (Fn. 4).

35 Vgl. Oscar W. Gabriel / Silke I. Keil, Kandidatenorientierungen in Teilelektoraten und Wahlverhalten, in: Hans Rattinger / Oscar W. Gabriel / Jürgen W. Falter (Hrsg.), Der gesamtdeutsche Wähler. Stabilität und Wandel des Wählerverhaltens im wiedervereinigten Deutschland, BadenBaden 2007, S. 357 - 381.

36 Vgl. zum Beispiel Richard R. Lau / David P. Redlaws, Advantages and Disadvantages of Cognitive Heuristics in Political Decision Making, in: American Journal of Political Science, 45. Jg. (2001), H. 4, S. 951 - 971; Jeffrey T. Mondak, Source Cues and Political Approval: The Cognitive Dynamics of Political Support for the Reagan Agenda, in: American Journal of Political Science, 37. Jg. (1993), H. 1, S. $186-212$.

37 Vgl. Oscar W. Gabriel / Jürgen Maier, Politische Soziologie, in: PVS, 50. Jg. (2009), H. 3 (im Druck).

38 Damit werden erste Ergebnisse aus einem breiter angelegten DFG-Projekt unter dem Titel „Politische Kenntnisse in der Bundesrepublik Deutschland: Verteilung, Struktur, Determinanten, Konsequenzen, 1949 - 2008“ veröffentlicht. 
es Wissensunterschiede hinsichtlich verschiedener inhaltlicher Bereiche? Zweitens, bestehen Differenzen in den politischen Kenntnissen von Ost- und Westdeutschen? Drittens, wie stabil ist das Wissen der Deutschen über die Zeit hinweg?

\section{Was ist politisches Wissen?}

Politisches Wissen wird in diesem Beitrag dem Vorschlag von Delli Carpini und Keeter folgend als „factual information about politics that is stored in long-term memory “39 definiert. Das zentrale Kriterium, um eine echte Wissensfrage von einer vermeintlichen zu trennen, ist das Vorhandensein einer objektiv richtigen Antwort. Dies ist zum Beispiel der Fall, wenn man danach fragt, welcher Partei der Bundeskanzler angehört, welche der beiden Stimmen bei der Bundestagswahl letztlich über die Stärke der Parteien im Bundestag entscheidet, oder wie viele Länder Mitglied der Europäischen Union sind.

Ausgeschlossen sind hingegen zum Beispiel Fragen zur Platzierung von Kandidaten, Gruppen oder Parteien auf ideologischen oder themenbezogenen Skalen. Hierbei gibt es kaum einen objektiven Maßstab, nach dem man eine Partei beispielsweise als „links“ oder „rechts“ einstufen könnte. Zwar können Rangordnungen aufgrund konsensuell festgelegter ideologischer Schemata oder Expertenratings gebildet werden; allerdings ist das Verständnis von ideologischen Positionen so unspezifisch, dass es äußerst schwer fällt, den „wahren“ Standpunkt eines Akteurs zu bestimmen. Ähnlich verhält es sich bei Fragen nach dem Bekanntheitsgrad von Politikern, der häufig als Abfallprodukt von Sympathiebewertungen nämlich über den Anteil derjenigen, die anstelle eines Ratings die „Weiß nicht“- beziehungsweise „Kenne ich nicht“-Kategorie wählen - ermittelt wird. Auch hier gibt es keine Möglichkeit, anhand eines externen Kriteriums zu bestimmen, ob die Frage korrekt beantwortet wurde (das heißt, ob Befragte, die den betreffenden Politiker nicht kennen, sich auch tatsächlich zu erkennen geben und nicht trotzdem eine Bewertung vornehmen, beziehungsweise ob Befragte, die den zu bewertenden Politiker kennen, auch tatsächlich ein Urteil über ihn fällen und nicht - aus welchen Gründen auch immer - mit „weiß nicht“ oder „kenne ich nicht“ antworten).

Neben dem eher technischen Problem, wie eine Wissensfrage gestellt sein muss, damit sie zuverlässig Aufschluss über die Kenntnisse von Befragten gibt, geht es bei der Erfassung von politischem Wissen aber auch immer darum, woran bemessen wird, ob entsprechende Kenntnisse vorliegen oder nicht. Allerdings gehen die Auffassungen darüber, welche politischen Fakten den Bürgern bekannt sein sollten, weit auseinander. Konsens besteht bestenfalls in der groben Absteckung des Gegenstandsbereiches. Im Anschluss an Barber ist man sich darüber einig, dass ein Bürger wissen sollte „what the government is and does“ ${ }^{40}$. Einerseits soll ein Individuum also über Wissen bezüglich der Strukturen des politischen Systems verfügen. Dieses gliedert sich in Kenntnisse über politische Institutionen und grundlegende Verfahrensweisen, zum Beispiel die Kenntnis des Wahlsystems. ${ }^{41}$ Andererseits wird

39 Vgl. Michael X. Delli Carpini / Scott Keeter, What Americans Know About Politics and Why it Matters, a.a.O. (Fn. 4), S. 10.

40 Vgl. James D. Barber, Citizen Politics, Chicago 1973, S. 44.

41 Vgl. W. Russell Neuman, a.a.O. (Fn. 4), S. 196. 
erwartet, dass ein Bürger über aktuelle politische Streitfragen informiert ist. ${ }^{42}$ Delli Carpini und Keeter betrachten darüber hinaus ein Minimum an Wissen über die zentralen politischen Akteure („who the government is") sowie Kenntnisse über fundamentale historische Zusammenhänge als essentiell. ${ }^{43}$ Wenngleich sie ein Standardinstrumentarium zur Messung von Politikkenntnissen vorschlagen ${ }^{44}$, besteht über die genauere inhaltliche Ausgestaltung der vier skizzierten Themenbereiche - also der Frage, mit welchen Items man die politische Expertise von Bürgern konkret messen kann - keinerlei Übereinkunft.

\section{Die politischen Kenntnisse der Bundesbürger: Datenlage}

Für den vorliegenden Beitrag wurden alle bei GESIS-ZA archivierten für die deutsche Bevölkerung repräsentativen Befragungen gesichtet, in denen auch Fragen zum politischen Wissen gestellt wurden. ${ }^{45}$ Für den Zeitraum von 1949 bis 2008 konnten insgesamt 114 Umfragen identifiziert werden, in denen mindestens eine Wissensfrage in dem hier definierten Sinn erhoben wurde. Zumeist handelt es sich um Querschnittsuntersuchungen; nur sechs Studien sind Wiederholungsbefragungen. ${ }^{46}$ Insgesamt finden sich 716 Fragen zum politischen Wissen, die im Folgenden die Grundgesamtheit bilden.

Die Erhebung politischer Kenntnisse in Meinungsumfragen wurde schon immer betrieben, was die nur spärliche Beschäftigung der politikwissenschaftlichen Forschung mit diesem Thema zusätzlich erklärungsbedürftig erscheinen lässt (siehe Abbildung 1). Selbst in Studien, die aus den Anfangsjahren der Bundesrepublik stammen, finden sich einige Fragen zum politischen Wissen der Deutschen. In den nachfolgenden Jahrzehnten wurde die Erfassung von Politikkenntnissen stetig intensiviert. Dies gilt insbesondere seit der deutschen Einheit. Für den Zeitraum 1990 bis 1999 liegen 268, für 2000 bis 2008200 Wissensfragen vor. ${ }^{47}$ Thematisch sind diese vorwiegend auf politische Institutionen und Prozesse ${ }^{48}$ (39,7 Prozent) und politische Inhalte ${ }^{49}$ (32,7 Prozent; vgl. Tabelle 1) gerichtet. Seltener wird die

42 Vgl. Bernard R. Berelson / Paul F. Lazarsfeld / William N. McPhee, a.a.O. (Fn. 3), S. 308.

43 Vgl. Michael X. Delli Carpini / Scott Keeter, Measuring Political Knowledge: Putting First Things First, in: American Journal of Political Science, 37. Jg. (1993), H. 4, S. 1179 - 1206, S. 1183.

44 Vgl. dies., What Americans Know About Politics and Why it Matters, a.a.O. (Fn. 4).

45 Stand vom Mai 2009. Nicht berücksichtigt wurden Studien, die ihren Fokus auf bestimmte Regionen (zum Beispiel Bundesländer, Kommunen) oder Populationen (zum Beispiel bestimmte Alters- oder Berufsgruppen) richten. Repräsentativität wurde nicht zwingend an die Verwendung von Zufallsstichproben geknüpft. In unserem Datensatz sind deshalb auch Umfragen enthalten, die - da mit dem Ziel durchgeführt, Aussagen für die Gesamtbevölkerung zu treffen - auf Quotenstichproben basieren.

46 Diese sind deshalb besonders interessant, weil sie die Analyse der zeitlichen Stabilität von politischem Wissen auf der Mikroebene ermöglichen (was aber nicht Gegenstand unseres Beitrags ist).

47 Es ist davon auszugehen, dass die Anzahl der Wissensfragen zwischen 2000 und 2008 unterschätzt wird. Zum einen ist das aktuelle Jahrzehnt noch nicht abgeschlossen. Streng genommen kann es also nicht mit früheren Zeitabschnitten verglichen werden. Zum anderen erfolgt die Bereitstellung von Daten durch GESIS-ZA zwangsläufig zeitverzögert, da deren Überstellung und Aufbereitung Zeit - zum Teil mehrere Jahre - in Anspruch nimmt.

48 Beispiel: „Welche Parteien bilden eigentlich heute in Bonn die Bundesregierung?“ (1967).

49 Beispiel: „Der Solidaritätszuschlag ist ein Zuschlag zur Einkommens- und Körperschaftsteuer. Wozu dient er?"“ (2008). 
Kenntnis politischer Akteure ${ }^{50}$ in den Blick genommen (22,8 Prozent). Kaum Beachtung finden Fragen aus den Bereichen politische Geographie und (Zeit-)Geschichte ${ }^{51}$ (3,8 Prozent). ${ }^{52}$ Allerdings sind dabei bestimmte Themenkonjunkturen zu erkennen. So stehen institutionelle Strukturen und Prozesse insbesondere in den Gründerjahren der Bundesrepublik sowie erneut seit 1990 im Mittelpunkt, wenn es um die Erhebung von politischem Wissen geht. Eine ähnliche Entwicklung ist für Fragen der politischen Geographie und der Zeitgeschichte zu erkennen. Die Kenntnis politischer Akteure war Thema der 1960er und 1970 er Jahre. Um politische Inhalte ging es vor allem in den 1980ern.



Aus methodischer Sicht überrascht, dass politisches Wissen überwiegend mit offenen Fragen erhoben wird (siehe Tabelle 2). Bis Ende der 1970er Jahre dominierten sie, und erst im aktuellen Jahrzehnt werden offene Fragen seltener eingesetzt als geschlossene. Dennoch sind sie nach wie vor populär: In rund einem Drittel aller Fälle entscheiden sich Primärforscher für sie und nicht für Fragen mit vorgegebenen Antwortkategorien.

Die in der Literatur sehr umstrittenen „Weiß nicht“-Kategorien werden bei etwa vier von zehn Wissensfragen verwendet. Bis Ende der 1970er Jahre wurde diese Form häufiger eingesetzt als Fragen ohne eine entsprechende Antwortoption. Seit den 1980er Jahren haben sich die Sichtweisen hier aber offensichtlich verändert, denn seither geht die Tendenz

50 Beispiel: „Können Sie mir sagen, wer gerade Außenminister ist?“ (1970).

51 Beispiel: „In Berlin gibt es ja die Mauer, die Ost- und Westberlin voneinander trennt. Können Sie mir sagen, in welchem Jahr diese Mauer gebaut wurde?" (1986).

52 Insgesamt acht Fragen (1,1 Prozent) konnten nicht klassifiziert werden. Dabei handelt es sich beispielsweise um die 1995 gestellte Frage, an welchem Tag der „Europatag“ gefeiert wird. 


\begin{tabular}{|l|c|c|c|c|c|c|c|}
\hline Tabelle 1: Verteilung der Wissensfragen nach Themenbereichen und Erhebungszeitraum (oberer \\
Wert: $N$; unterer Wert: Spaltenprozente) \\
\hline \multirow{2}{*}{ Themenbereich } & \multicolumn{7}{|c|}{ Erhebungszeitraum } \\
\cline { 2 - 8 } & Gesamt & $\begin{array}{c}1949- \\
1959\end{array}$ & $\begin{array}{c}1960- \\
1969\end{array}$ & $\begin{array}{c}1970- \\
1979\end{array}$ & $\begin{array}{c}1980- \\
1989\end{array}$ & $\begin{array}{c}1990- \\
1999\end{array}$ & $\begin{array}{c}2000- \\
2008\end{array}$ \\
\hline Politische Institutionen & 284 & 8 & 2 & 24 & 11 & 152 & 87 \\
und Prozesse & $(39,7)$ & $(38,1)$ & $(4,2)$ & $(33,3)$ & $(10,3)$ & $(56,7)$ & $(43,5)$ \\
& 163 & 5 & 45 & 29 & 1 & 50 & 33 \\
Politische Akteure & $(22,8)$ & $(23,8)$ & $(93,8)$ & $(40,3)$ & $(0,9)$ & $(18,7)$ & $(16,5)$ \\
& 234 & 6 & - & 18 & 93 & 50 & 67 \\
Politische Inhalte & $(32,7)$ & $(28,6)$ & $(-)$ & $(25,0)$ & $(86,9)$ & $(18,7)$ & $(33,5)$ \\
Politische Geographie & 27 & 2 & 1 & 1 & 2 & 11 & 10 \\
und Geschichte & $(3,8)$ & $(9,5)$ & $(2,1)$ & $(1,4)$ & $(1,9)$ & $(4,1)$ & $(5,0)$ \\
Sonstiges & 8 & - & - & - & - & 5 & 3 \\
& $(1,1)$ & $(-)$ & $(-)$ & $(-)$ & $(-)$ & $(1,9)$ & $(1,5)$ \\
\hline Gesamt & 716 & 21 & 48 & 72 & 107 & 268 & 200 \\
\hline Quelle: Eigene Zusammenstellung auf Basis verschiedener Studien des GESIS-Datenarchivs. \\
\hline
\end{tabular}

\begin{tabular}{|c|c|c|c|c|c|c|c|}
\hline $\begin{aligned} \text { Tabelle 2: } & \text { Verteilung } \\
& \text { Wert: } N ; \mathrm{u}\end{aligned}$ & $\begin{array}{l}\text { Wissensf } \\
r \text { Wert: }\end{array}$ & $\begin{array}{l}\text { gen nac } \\
\text { Daltenpr }\end{array}$ & $\begin{array}{l}\text { rageme } \\
\text { ente) }\end{array}$ & alen $u$ & Erhebu & tran & berer \\
\hline & & & & bungszeit & $\mathrm{um}$ & & \\
\hline Themenbereich & Gesamt & $\begin{array}{l}1949- \\
1959\end{array}$ & $\begin{array}{l}1960- \\
1969\end{array}$ & $\begin{array}{l}1970- \\
1979\end{array}$ & $\begin{array}{l}1980- \\
1989\end{array}$ & $\begin{array}{l}1990- \\
1999\end{array}$ & $\begin{array}{l}2000- \\
2008\end{array}$ \\
\hline Fragetyp & & & & & & & \\
\hline offen & $\begin{array}{c}394 \\
(55,0)\end{array}$ & $\begin{array}{c}20 \\
(95,2)\end{array}$ & $\begin{array}{c}33 \\
(68,8)\end{array}$ & $\begin{array}{c}71 \\
(98,6)\end{array}$ & $\begin{array}{c}71 \\
(66,4)\end{array}$ & $\begin{array}{c}136 \\
(50,7)\end{array}$ & $\begin{array}{c}63 \\
(31,5)\end{array}$ \\
\hline geschlossen & $\begin{array}{c}322 \\
(45,0)\end{array}$ & $\begin{array}{c}1 \\
(4,8)\end{array}$ & $\begin{array}{c}15 \\
(31,2)\end{array}$ & $\begin{array}{c}1 \\
(1,4)\end{array}$ & $\begin{array}{c}36 \\
(33,6)\end{array}$ & $\begin{array}{c}132 \\
(49,3)\end{array}$ & $\begin{array}{c}137 \\
(68,5)\end{array}$ \\
\hline "Weiß nicht"-Kategorie & & & & & & & \\
\hline $\mathrm{Ja}$ & $\begin{array}{c}300 \\
(41,9)\end{array}$ & $\begin{array}{c}11 \\
(52,4)\end{array}$ & $\begin{array}{c}30 \\
(62,5)\end{array}$ & $\begin{array}{c}50 \\
(69,4)\end{array}$ & $\begin{array}{c}44 \\
(41,1)\end{array}$ & $\begin{array}{c}78 \\
(29,1)\end{array}$ & $\begin{array}{c}87 \\
(43,5)\end{array}$ \\
\hline Nein & $\begin{array}{c}416 \\
(59,1)\end{array}$ & $\begin{array}{c}10 \\
(47,6)\end{array}$ & $\begin{array}{c}18 \\
(37,5)\end{array}$ & $\begin{array}{c}22 \\
(30,6)\end{array}$ & $\begin{array}{c}63 \\
(58,9)\end{array}$ & $\begin{array}{c}190 \\
(70,9)\end{array}$ & $\begin{array}{c}113 \\
(56,5)\end{array}$ \\
\hline Erhebungsmodus & & & & & & & \\
\hline persönlich & $\begin{array}{c}565 \\
(78,9)\end{array}$ & $\begin{array}{c}21 \\
(100,0)\end{array}$ & $\begin{array}{c}48 \\
(100,0)\end{array}$ & $\begin{array}{c}72 \\
(100,0)\end{array}$ & $\begin{array}{c}107 \\
(100,0)\end{array}$ & $\begin{array}{c}231 \\
(86,2)\end{array}$ & $\begin{array}{c}86 \\
(43,0)\end{array}$ \\
\hline telefonisch & $\begin{array}{c}151 \\
(21,1)\end{array}$ & $\begin{array}{l}- \\
(-)\end{array}$ & $\begin{array}{l}- \\
(-)\end{array}$ & $\frac{-}{(-)}$ & $\begin{array}{l}- \\
(-)\end{array}$ & $\begin{array}{c}37 \\
(13,8)\end{array}$ & $\begin{array}{c}114 \\
(57,0)\end{array}$ \\
\hline Gesamt & 716 & 21 & 48 & 72 & 107 & 268 & 200 \\
\hline
\end{tabular}


eindeutig zu Formulierungen, in denen die Befragten nicht die Möglichkeit erhalten, ihr fehlendes Wissen angemessen zu erkennen zu geben. Dies hat für die Erforschung von politischen Kenntnisständen weitreichende Implikationen, da man bei Fragen dieses Typs nicht mehr in der Lage ist, Fehlinformierte (das heißt Personen, die sich für eine falsche Antwortalternative entscheiden) von Uninformierten (das heißt Personen, die die „Weiß nicht“-Kategorie wählen) zu trennen. Der Trend hin zu mehr geschlossenen Fragen und die Tendenz, Befragten die „Weiß nicht“-Kategorie nicht anzubieten, hängt möglicherweise mit Veränderungen in der Art und Weise zusammen, wie Informationen über das Politikwissen der Deutschen gesammelt werden. Jedenfalls ist zu beobachten, dass repräsentative Studien, die auch Fragen zu politischen Kenntnissen beinhalten, gegenwärtig überwiegend telefonisch durchgeführt werden. Noch bis Ende der 1980er Jahre fanden diese Befragungen ausschließlich persönlich statt.

\section{Die politischen Kenntnisse der Bundesbürger: Ausgewählte Ergebnisse}

Um das Wissen der Deutschen über Politik zu bestimmen, wurde für alle 716 Fragen der Anteil der korrekten Antworten - also des objektiv vorhandenen Faktenwissens - ermittelt. Unabhängig vom Inhalt der gestellten Fragen und vom Erhebungszeitpunkt zeigt sich, dass der Median insgesamt bei Md = 44,1 liegt. In Abbildung 2 sind die Mediane als schwarze Querstriche dargestellt, die die jeweilige Fragemenge in eine „obere“ Hälfte, das heißt Fragen, zu denen überdurchschnittlich viel Wissen vorhanden ist, und eine „untere“ Hälfte, das heißt Fragen, zu denen die Bürger unterdurchschnittlich viel wissen, einteilt. Dass die Deutschen kaum über politische Kenntnisse verfügen, lässt sich damit empirisch ebenso wenig belegen wie das Ideal des gut informierten Bürgers. Vielmehr liegt die Wahrheit ziemlich genau in der Mitte. Ähnlich wie in den Vereinigten Staaten gilt also auch in Deutschland: „Although citizens are not fully informed, they are not fully ignorant." 53

\subsection{Themenspezifische Kenntnisstände}

Differenziert man alle in den Umfragen identifizierten Wissensfragen nach Themenbereichen, zeigt sich, dass es den Bundesbürgern am leichtesten fällt, Fragen zu den politischen Akteuren $(\mathrm{Md}=58,6)$ und den strukturellen Merkmalen des politischen Systems $(\mathrm{Md}=50,0)$ zu beantworten. Dies erscheint plausibel, denn einerseits sind die politischen Akteure, insbesondere die im Bundestag vertretenen Parteien und ihr Spitzenpersonal, besonders sichtbar. Andererseits handelt es sich bei Kenntnissen über politische Institutionen und Prozesse um Wissen, das in der Schule vermittelt und - etwa im Rahmen von Klassenarbeiten - dort auch überprüft wird. Zudem werden zentrale institutionelle Strukturen nur selten verändert, so dass die Chance vergleichsweise groß ist, einmal erlerntes Wissen über einen längeren Zeitraum zu erhalten. Den dritten Platz nehmen Fragen zu Geographie und Geschichte ein $(\mathrm{Md}=44,5)$. Abgeschlagen sind Kenntnisse über politische Inhalte $(\mathrm{Md}=30,7)$ - also über den Bereich der Politik, der sich zum einen ständig wandelt, zum

53 Michael X. Delli Carpini / Scott Keeter, What Americans Know About Politics and Why it Matters, a.a.O. (Fn. 4), S. 98. 
anderen besonders kompliziert und undurchsichtig ist. Gliedert man die Inhalte nach Politikbereichen auf, so zeigen sich allerdings deutliche und statistisch signifikante Unterschiede $(H=38,33 ; p \leq 0,001)$. Die größten Kenntnisse bestehen interessanterweise auf dem Feld der europäischen Politik ( $\mathrm{Md}=41,2)$, das allgemeinhin als besonders schwer durchschaubar gilt. Demgegenüber ist das Wissen über bundespolitische Themen vergleichsweise gering $(M=36,8)$. Am weitaus schlechtesten schneiden die Deutschen bei Fragen zur internationalen Politik ab $(\mathrm{Md}=7,8)$.



Ein zweiter Blick auf Abbildung 2 zeigt, dass durchaus Themen existieren, zu denen nahezu jeder die korrekte Antwort weiß. So etwa identifizierten 1993 99,4 Prozent, dass die Ukraine kein Mitglied der Europäischen Union ist. Ebenso gelang es 2000 96,7 Prozent, Bundeskanzler Gerhard Schröder der richtigen Partei zuzuordnen. Es gibt aber auch politische Fragen, die kaum jemand richtig beantworten kann: Beispielsweise wussten im Frühjahr 1999 nur 8,3 Prozent, an welchem Tag, die kommenden Wahlen zum Europäischen Parlament stattfinden. Ebenfalls kaum bekannt sind die Abgeordneten des Europäischen Parlaments. 1993 gelang es nur 4,8 Prozent, ein deutsches Mitglied zu benennen. Die enorme Spannweite, die im Wissen der Deutschen besteht, indizieren die Querstriche am Ende der dünnen Linien, die jeweils die Frage repräsentieren, zu der die meisten und die wenigsten 
Kenntnisse vorliegen. Dieser Eindruck wird unterstrichen, wenn man neben Minimum und Maximum auch die Streuung der richtigen Antworten um den Median ausweist. Je länger der etwas breitere, grau eingefärbte Balken ist, desto heterogener ist das Wissen verteilt. ${ }^{54}$ Die Differenzierung nach Themenbereichen belegt, dass politische Kenntnisse in der Bevölkerung durch die Bank weg stark ungleich verteilt sind.

\subsection{Wissensunterschiede zwischen Ost- und Westdeutschland?}

In der Literatur wurden insbesondere zu Beginn der 1990er Jahre Wissensunterschiede zwischen Ost- und Westdeutschen berichtet. Die hierfür angebotene Erklärung, dass die Bürger in den neuen Bundesländern über weniger Politikkenntnisse verfügen, weil ihnen die Erfahrung im Umgang mit institutionellen Strukturen, politischen Inhalten und politischen Akteuren fehlt, scheint durchaus plausibel. Dazu passt einerseits, dass Ostdeutsche über Themen und Akteure, die eine enge Beziehung zur Lebenswelt der ehemaligen DDR aufweisen, deutlich mehr Wissen vorweisen können als die Bürger in den alten Bundesländern. Andererseits scheinen die Unterschiede mit zunehmender Entfernung zur deutschen Einheit zu verschwinden. Allerdings stützen sich diese Befunde durchweg nur auf sehr wenige Indikatoren zum politischen Faktenwissen, können also nicht ohne Weiteres generalisiert werden.

Ausweislich der hier vorgelegten Daten ist die bislang in der Literatur vorherrschende Einschätzung nicht belastbar. So zeigt sich insgesamt, dass die Wissensunterschiede zwischen Ost- und Westdeutschen kaum ins Gewicht fallen (siehe Tabelle 3). Dies gilt nicht nur für den Zeitraum von 2000 bis 2008, sondern auch für die ersten Jahre nach der deutschen Einheit. ${ }^{55}$ Auch wenn man nach Themenbereichen differenziert, ergeben sich nur wenige Ost-West-Differenzen. Diese fallen hinsichtlich der Kenntnis politischer Akteure noch am größten aus, sind aber auch hier - wie hinsichtlich aller anderen Fragegegenstände - nicht signifikant. ${ }^{56}$

\subsection{Stabilität und Wandel des politischen Wissens}

Die langfristigen Entwicklungen politischer Kenntnisse zu untersuchen, stellt aus mehreren Gründen eine besondere Herausforderung dar: Erstens variieren die gestellten Fragen in Frage- und Antwortformat sehr stark; zweitens wird Wissen meist nur sehr punktuell abgefragt; drittens gibt es kaum lange Zeitreihen. Konkret konnten hier 15 Wissensfragen iden-

54 Genau genommen weist das untere Ende des Balkens das 25. Perzentil und das obere Ende des Balkens das 75. Perzentil der Verteilung aus. Die Differenz zwischen den beiden Perzentilen kann als Maß für die Streuung der Messwerte interpretiert werden, da sie den Bereich definiert, in dem sich die mittleren 50 Prozent der Messwerte befinden.

55 Dies schließt natürlich nicht aus, dass sich die Kenntnisstände in Bezug auf Einzelfragen in den alten und den neuen Bundesländern voneinander unterscheiden. Ein genereller Ost-West-Unterschied lässt sich aber nicht beobachten.

56 Der Medianunterschied zwischen Ost- und Westdeutschland ist nicht signifikant. 1990 bis 1999: $\mathrm{z}=-0,254, \mathrm{p}>0,05 ; 2000$ bis 2008: $\mathrm{z}=-0,319, \mathrm{p}>0,05$ (Mann-Whitney-U-Test), für einzelne Themenbereiche alle $\mathrm{z}<1,00, \mathrm{p}>0,05$. 


\begin{tabular}{|c|c|c|c|c|}
\hline \multicolumn{5}{|c|}{$\begin{array}{l}\text { Tabelle 3: Anteil richtiger Antworten in alten und neuen Bundesländern (ABL, NBL) nach } \\
\text { Themengebiet und Erhebungszeitraum (Median) }\end{array}$} \\
\hline \multirow{2}{*}{ Themenbereich } & \multicolumn{2}{|c|}{1990 bis 1999} & \multicolumn{2}{|c|}{2000 bis 2008} \\
\hline & $\mathrm{ABL}$ & NBL & $\mathrm{ABL}$ & NBL \\
\hline $\begin{array}{l}\text { Politische Institutionen } \\
\text { und Prozesse }\end{array}$ & $\begin{array}{c}73,9 \\
(\mathrm{~N}=92)\end{array}$ & $\begin{array}{c}71,4 \\
(\mathrm{~N}=91)\end{array}$ & $\begin{array}{c}53,2 \\
(\mathrm{~N}=33)\end{array}$ & $\begin{array}{c}55,5 \\
(\mathrm{~N}=33)\end{array}$ \\
\hline Politische Akteure & $\begin{array}{c}56,4 \\
(\mathrm{~N}=40)\end{array}$ & $\begin{array}{c}61,4 \\
(\mathrm{~N}=38)\end{array}$ & $\begin{array}{c}60,3 \\
(\mathrm{~N}=5)\end{array}$ & $\begin{array}{c}52,5 \\
(\mathrm{~N}=5)\end{array}$ \\
\hline Politische Inhalte & $\begin{array}{c}36,7 \\
(\mathrm{~N}=48)\end{array}$ & $\begin{array}{c}34,2 \\
(\mathrm{~N}=48)\end{array}$ & $\begin{array}{c}47,6 \\
(N=65)\end{array}$ & $\begin{array}{c}46,0 \\
(\mathrm{~N}=65)\end{array}$ \\
\hline Politische Inhalte: Bundesebene & $(-)$ & $(-)$ & $\begin{array}{c}43,1 \\
(\mathrm{~N}=20)\end{array}$ & $\begin{array}{c}42,3 \\
(\mathrm{~N}=20)\end{array}$ \\
\hline $\begin{array}{l}\text { Politische Inhalte: } \\
\text { Europäische Ebene }\end{array}$ & $\begin{array}{c}36,7 \\
(\mathrm{~N}=48)\end{array}$ & $\begin{array}{c}34,2 \\
(\mathrm{~N}=48)\end{array}$ & $\begin{array}{c}49,4 \\
(\mathrm{~N}=45)\end{array}$ & $\begin{array}{c}46,8 \\
(N=45)\end{array}$ \\
\hline $\begin{array}{l}\text { Politische Inhalte: Internationale } \\
\text { Ebene }\end{array}$ & $(-)$ & $(-)$ & $(-)$ & $(-)$ \\
\hline $\begin{array}{l}\text { Politische Geographie } \\
\text { und Geschichte }\end{array}$ & $\begin{array}{c}42,4 \\
(\mathrm{~N}=10)\end{array}$ & $\begin{array}{c}44,0 \\
(\mathrm{~N}=10)\end{array}$ & $\begin{array}{c}44,6 \\
(\mathrm{~N}=9)\end{array}$ & $\begin{array}{c}47,5 \\
(\mathrm{~N}=9)\end{array}$ \\
\hline Sonstiges & $\begin{array}{c}5,9 \\
(\mathrm{~N}=5) \\
\end{array}$ & $\begin{array}{c}2,4 \\
(\mathrm{~N}=5) \\
\end{array}$ & $(-)$ & $(-)$ \\
\hline Gesamt & $\begin{array}{c}53,4 \\
(\mathrm{~N}=195)\end{array}$ & $\begin{array}{c}53,5 \\
(\mathrm{~N}=192)\end{array}$ & $\begin{array}{c}49,7 \\
(\mathrm{~N}=112)\end{array}$ & $\begin{array}{c}48,3 \\
(\mathrm{~N}=112)\end{array}$ \\
\hline
\end{tabular}

tifiziert werden, die zu zwei Zeitpunkten gestellt wurden, fünf Fragen, die dreimal und sechs, die mehr als dreimal erhoben wurden. Die mit Abstand längste Zeitreihe liegt für die Kenntnis des Wahlsystems vor; danach wurde seit 1980 zwölfmal ohne nennenswerte Änderungen des Wortlauts oder des Fragetyps gefragt. Mit Ausnahme der Erhebung des Jahres 1990 zeigt sich hier eine enorme Stabilität des Wissens; der Anteil korrekter Antworten pendelt stets um die 50-Prozentmarke (siehe Abbildung 3).

Selbst wenn es einige wenige Fragen gibt, die in den letzten fast sechs Jahrzehnten mehrfach gestellt wurden, ist die Basis, auf der man über Stabilität und Wandel von politischem Wissen in Deutschland urteilen kann, doch sehr klein. Aus diesem Grund haben wir uns entschieden, die korrekten Antworten auf alle verfügbaren Wissensfragen zusammenzufassen und die Entwicklung des Medians über den gesamten Beobachtungszeitraum zu untersuchen. Dabei fällt auf, dass das Wissensniveau über die Jahre hinweg beträchtlich schwankt (siehe Abbildung 4). Während der Kenntnisstand der Deutschen in den ersten beiden Jahrzehnten nach Gründung der Bundesrepublik ausgehend von einem moderaten Niveau deutlich angewachsen war, fiel er in den 1970er und 1980er Jahren erheblich ab. In den beiden darauffolgenden Jahrzehnten sind die Kenntnisse wieder stark gestiegen. Auch wenn die Ergebnisse nicht unmittelbar miteinander vergleichbar sind (schließlich wurden in den unterschiedlichen Zeitperioden unterschiedliche Fragen gestellt, die zudem mit einer unter- 


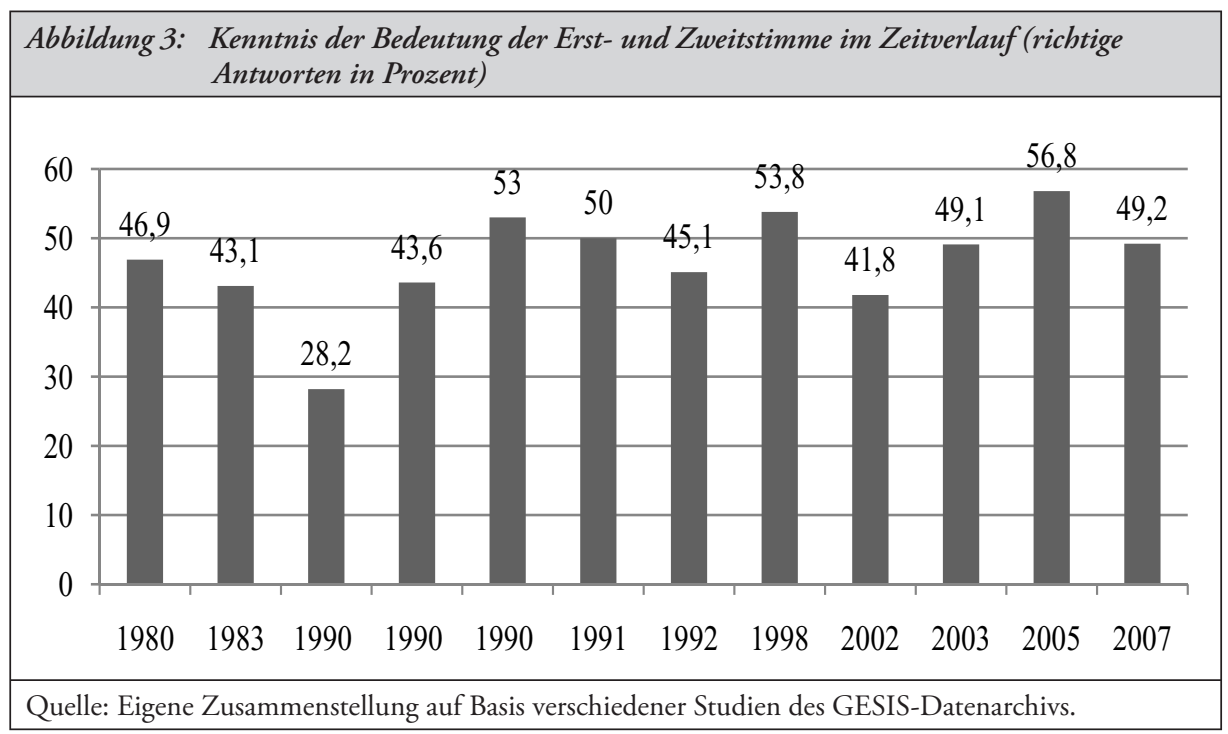

schiedlichen Methodik ${ }^{57}$ erhoben wurden), ist zumindest auf den ersten Blick von einem universellen Anstieg politischen Wissens nichts zu erkennen - und zwar obwohl sich das Bildungsniveau und die Möglichkeiten, auf politische Informationen zuzugreifen, in den letzten Jahrzehnten deutlich verbessert haben. Lässt man die 1970er und 1980er Jahre außer Acht, kann man eher von einer weitgehenden Stabilität sprechen. Dieses Ergebnis steht in scharfem Kontrast zur positiven Entwicklung anderer Größen der politischen Involvierung in diesem Zeitraum wie zum Beispiel dem politischen Interesse oder dem subjektiven politischen Kompetenzgefühl. ${ }^{58}$

Eine Differenzierung nach Themenbereichen zeigt, dass über alle Wissensgebiete starke Variationen über die Zeit hinweg zu beobachten sind, ohne dass ein eindeutiger Trend zu erkennen ist (siehe Tabelle 4). So schwankt der Anteil der richtigen Antworten zu den politischen Institutionen und Prozessen zwischen knapp 40 und 69 Prozent. Noch größer ist die Bandbreite für politische Akteure, wo sich die entsprechenden Werte zwischen rund 16 und 78 Prozent bewegen, sowie für politische Inhalte, deren Kenntnis zwischen einem und 48 Prozent variiert. Zu politischer Geographie und Zeitgeschichte sind erst in den letzten beiden Jahrzehnten in ausreichendem Maß Wissensfragen gestellt worden; im Mittel bewegen sich die Kenntnisse hier jeweils knapp über 40 Prozent.

57 Weiterführende Analysen zeigen, dass der Fragetyp einen erheblichen Einfluss auf das gemessene Wissensniveau hat. So liegt der ermittelte Kenntnisstand bei offenen Fragen erheblich unter dem geschlossener Fragen (Md = 16,6 vs. Md = 64,3; Mann-Whitney-U-Test: $\mathrm{z}=-13,24, \mathrm{p} \leq$ 0,001). Allerdings muss dabei beachtet werden, dass Personen, die eine geschlossene Frage korrekt beantworten, nicht zwingend auch die richtige Antwort wissen. Da die Möglichkeit besteht, auch durch Raten die richtige Antwort zu wählen, werden Kenntnisstände mit geschlossenen Fragen systematisch überschätzt. Vgl. Michael X. Delli Carpini / Scott Keeter, What Americans Know About Politics and Why it Matters, a.a.O. (Fn. 4), S. $65-66$.

58 Vgl. Thorsten Faas / Jürgen Maier / Andreas Wüst, Wahlen und Wähler in der Bundesrepublik. Eine Einführung, Wiesbaden 2009 (im Erscheinen). 


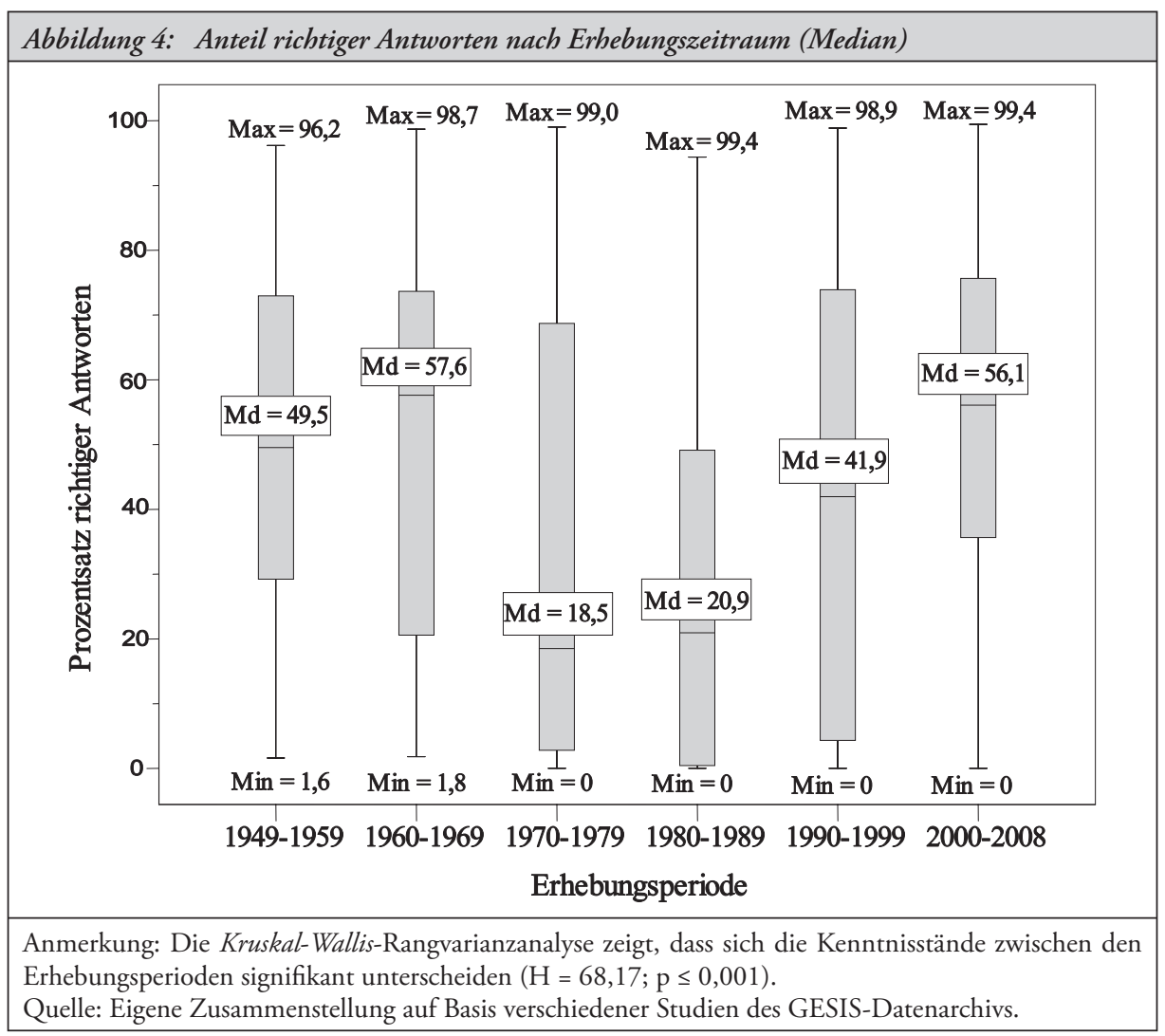

\section{Politisches Wissen: Erste Befunde und viele offene Fragen}

Mit Blick auf das politische Wissen ist zwischenzeitlich gut belegt, dass zwischen dem Ideal des gut informierten - und damit prinzipiell zu „rationalen“ Entscheidungen fähigen Bürgers - und der Realität eine erhebliche Diskrepanz besteht. Dies gilt insbesondere für die Vereinigten Staaten, aber auch für die Bundesrepublik Deutschland. Im Unterschied zu den USA, die auf eine breite Forschung auf diesem Gebiet zurückblicken können, liegen nur einige wenige Untersuchungen zum politischen Wissen der Bundesbürger vor. In Anbetracht der Konzentration auf den Zeitraum nach 1990, der Verwendung von Daten, die in aller Regel im Umfeld von Bundestagswahlen erhoben wurden, und der Fokussierung auf einige wenige Wissensfragen ist es allerdings fraglich, inwieweit die darin berichteten Ergebnisse verallgemeinert werden können.

Um die empirische Basis zu vergrößern, auf der Aussagen über das politische Wissen in Deutschland getroffen werden können, wurden - gewissermaßen als "Kassensturz“ - alle bei GESIS-ZA verfügbaren Umfragen ausgewertet, die auch Wissensfragen zu politischen Themen beinhalten. Die Ergebnisse lassen sich wie folgt zusammenfassen:

(1) Die Ausblendung der Frage, wie viel die Deutschen über Politik wissen, kann nicht mit dem Fehlen entsprechender Daten begründet werden. Für den Zeitraum von 1949 bis 


\begin{tabular}{|c|c|c|c|c|c|}
\hline \multirow[b]{2}{*}{ Erhebungszeitraum } & \multicolumn{5}{|c|}{ Themenbereich } \\
\hline & $\begin{array}{c}\text { Politische } \\
\text { Institutionen } \\
\text { und Prozesse }\end{array}$ & $\begin{array}{c}\text { Politische } \\
\text { Akteure }\end{array}$ & $\begin{array}{l}\text { Politische } \\
\text { Inhalte }\end{array}$ & $\begin{array}{c}\text { Politische } \\
\text { Geographie } \\
\text { und Geschichte }\end{array}$ & Sonstiges \\
\hline 1949 bis 1959 & $\begin{array}{c}45,3 \\
(\mathrm{~N}=8)\end{array}$ & $\begin{array}{c}48,5 \\
(\mathrm{~N}=5)\end{array}$ & $\begin{array}{c}45,7 \\
(N=6)\end{array}$ & $\begin{array}{c}76,1 \\
(\mathrm{~N}=2)\end{array}$ & - \\
\hline 1960 bis 1969 & $\begin{array}{c}60,0 \\
(\mathrm{~N}=2)\end{array}$ & $\begin{array}{c}20,0 \\
(\mathrm{~N}=45)\end{array}$ & - & $\begin{array}{c}52,7 \\
(\mathrm{~N}=1)\end{array}$ & - \\
\hline 1970 bis 1979 & $\begin{array}{c}69,0 \\
(\mathrm{~N}=24)\end{array}$ & $\begin{array}{c}16,8 \\
(\mathrm{~N}=29)\end{array}$ & $\begin{array}{c}0,7 \\
(\mathrm{~N}=18)\end{array}$ & $\begin{array}{c}3,9 \\
(\mathrm{~N}=1)\end{array}$ & - \\
\hline 1980 bis 1989 & $\begin{array}{c}51,4 \\
(\mathrm{~N}=11)\end{array}$ & $\begin{array}{c}16,4 \\
(\mathrm{~N}=1)\end{array}$ & $\begin{array}{c}9,6 \\
(\mathrm{~N}=93)\end{array}$ & $\begin{array}{c}46,4 \\
(\mathrm{~N}=2)\end{array}$ & - \\
\hline 1990 bis 1999 & $\begin{array}{c}39,7 \\
(\mathrm{~N}=152)\end{array}$ & $\begin{array}{c}60,6 \\
(\mathrm{~N}=50)\end{array}$ & $\begin{array}{c}35,5 \\
(\mathrm{~N}=50)\end{array}$ & $\begin{array}{c}42,1 \\
(\mathrm{~N}=11)\end{array}$ & $\begin{array}{c}4,8 \\
(\mathrm{~N}=5)\end{array}$ \\
\hline 2000 bis 2008 & $\begin{array}{c}56,8 \\
(\mathrm{~N}=87)\end{array}$ & $\begin{array}{c}77,7 \\
(\mathrm{~N}=33)\end{array}$ & $\begin{array}{c}48,1 \\
(\mathrm{~N}=67)\end{array}$ & $\begin{array}{c}44,0 \\
(\mathrm{~N}=10)\end{array}$ & $\begin{array}{c}47,4 \\
(\mathrm{~N}=3)\end{array}$ \\
\hline Gesamt & $\begin{array}{c}50,0 \\
(\mathrm{~N}=284)\end{array}$ & $\begin{array}{c}58,6 \\
(\mathrm{~N}=163)\end{array}$ & $\begin{array}{c}30,7 \\
(\mathrm{~N}=234)\end{array}$ & $\begin{array}{c}44,5 \\
(\mathrm{~N}=27)\end{array}$ & $\begin{array}{c}6,2 \\
(\mathrm{~N}=8)\end{array}$ \\
\hline
\end{tabular}

2008 konnten insgesamt 114 Umfragestudien mit 716 politischen Wissensfragen zu verschiedenen Themenbereichen identifiziert werden. Allerdings ist die Qualität dieser Daten begrenzt: Nur wenige der dokumentierten Fragen sind wiederholt gestellt worden; zudem variieren die eingesetzten Fragetypen stark.

(2) Die verbreitete Vorstellung vom weitgehend uninformierten Bürger kann nicht aufrechterhalten werden. Dazu wissen die Deutschen zu viel über Politik. Ebenso muss die Wunschvorstellung vom wohlinformierten Bürger verworfen werden - dazu ist das gemessene Wissensniveau zu niedrig. Allerdings hängen die gefundenen Ergebnisse stark vom Inhalt der gestellten Fragen und der dabei zur Anwendung kommenden Methodik ab. Beleg hierfür ist die enorme Streuung der Kenntnisstände: So gibt es einerseits zahlreiche Fragen, die von fast allen beantwortet werden können, andererseits auch solche, auf die praktisch niemand eine Antwort weiß. Ebenso gibt es Effekte des Frageformats.

(3) Generelle Wissensunterschiede zwischen Ost- und Westdeutschen liegen nicht vor - und zwar weder für bestimmte Zeiträume noch für bestimmte Themen.

(4) Die politischen Kenntnisse der Deutschen sind über die Zeit hinweg weitgehend stabil. Ausnahmen sind die 1970er und 1980er Jahre, in denen das Wissensniveau erheblich niedriger lag als in früheren oder späteren Zeiträumen. Wie dieser Befund erklärt werden kann, müssen weiterführende Analysen zeigen, die sich nicht nur auf die Aggregatebene beschränken können.

Die ersten Ergebnisse aus dem oben genannten Projekt machen deutlich, dass sich die Beschäftigung mit diesem Thema durchaus lohnt - nicht zuletzt, um den Anschluss an die internationale politikwissenschaftliche Forschung zu halten, die sich schon seit geraumer Zeit mit der Bedeutung von Kognitionen für politische Einstellungen und politisches Ver- 
halten beschäftigt. Nachdem die Politische Soziologie in Deutschland hier einen enormen Nachholbedarf hat, stellen sich für die Zukunft vielfältige Fragen: Warum variiert das Wissen (zwischen Themen, aber auch zwischen Bürgern) so stark? Warum kann kein Anwachsen von Kenntnissen beobachtet werden, obwohl sich die politischen, sozialen und technologischen Rahmenbedingungen in den letzten Jahrzehnten dramatisch geändert haben? Durch welche Größen auf der Mikro- (zum Beispiel Sozialstruktur, Einstellungen, Mediennutzungsverhalten, Vorwissen) und der Makroebene (zum Beispiel Informationsumwelten, Systemstrukturen) wird das Politikwissen beeinflusst? Und welche Rolle spielen die bei der Messung zum Einsatz kommenden Erhebungsmethoden? Welche Konsequenzen ergeben sich zum Beispiel für persönliche Überzeugungssysteme, individuelles politisches Verhalten, aber auch für demokratische Systeme, wenn Bürger politische Experten oder politische Novizen sind? Last but not least: Welche Fragen eignen sich eigentlich im Rahmen von Befragungen, um zuverlässig auf das - selbstverständlich viel umfassendere - politische Wissen von Bürgern zu schließen - und was sagen die Ergebnisse solcher Wissenstests darüber aus, was Bürger von Politik tatsächlich verstehen?

\title{
Die Strategie der kommunizierten Ehrlichkeit im CDU/CSU-Bundestagswahlkampf 2005
}

\author{
Volker Best
}

Nach der Neuwahlankündigung durch Bundeskanzler Gerhard Schröder und SPD-Parteichef Franz Müntefering am 22. Mai 2005 musste der folgende Bundestagswahlkampf "gleichsam aus dem Boden gestampft werden"1. Umso bemerkenswerter scheint, dass ausgerechnet dieser Wahlkampf eine Innovation hervorbrachte: die Strategie der kommunizierten Ehrlichkeit von CDU und CSU, die im Folgenden hinsichtlich ihrer Motivation und Ausgestaltung, ihrer Probleme und Effekte analysiert werden soll.

\section{Strategien im Wahlkampf der Union 2005}

Die Ehrlichkeitsstrategie kann nicht isoliert von den anderen Wahlkampfstrategien der Union betrachtet werden. Fünf sind hier aus Sicht des Verfassers zu identifizieren ${ }^{2}$ : Es sollte erstens ein detailliertes Regierungsprogramm vorgelegt werden (gouvernementaler Wahlkampf ${ }^{3}$ ). Zweitens sollte sich der Wahlkampf an Themen statt an Personen orientieren

1 Christina Holtz-Bacha, Bundestagswahl 2005 - Die Überraschungswahl, in: dies. (Hrsg.), Die Massenmedien im Wahlkampf. Die Bundestagswahl 2005, Wiesbaden 2006, S. 5 - 31, S. 5.

2 Wahlkämpfe sind Bestandteil des Arkanbereichs von Politik; daher sind Kampagnenziele und -entscheidungen empirisch nicht einfach zu untersuchen. Vgl. Harald Schoen, Wahlkampfforschung, in: ders. / Jürgen W. Falter (Hrsg.), Handbuch Wahlforschung, Wiesbaden 2005, S. 503 -542, S. 505.

3 Dieser Begriff stammt von Frank Brettschneider, Bundestagswahlkampf und Medienberichterstattung, in: APuZ, B $51 / 52$ (2005), S. 19 - 26, S. 22. 